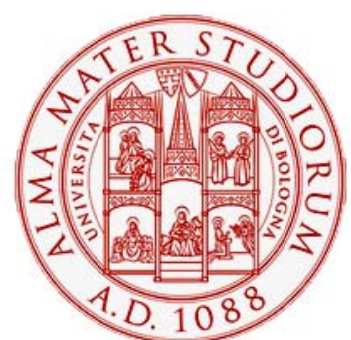

Alma Mater Studiorum - Università di Bologna DEPARTMENT OF ECONOMICS

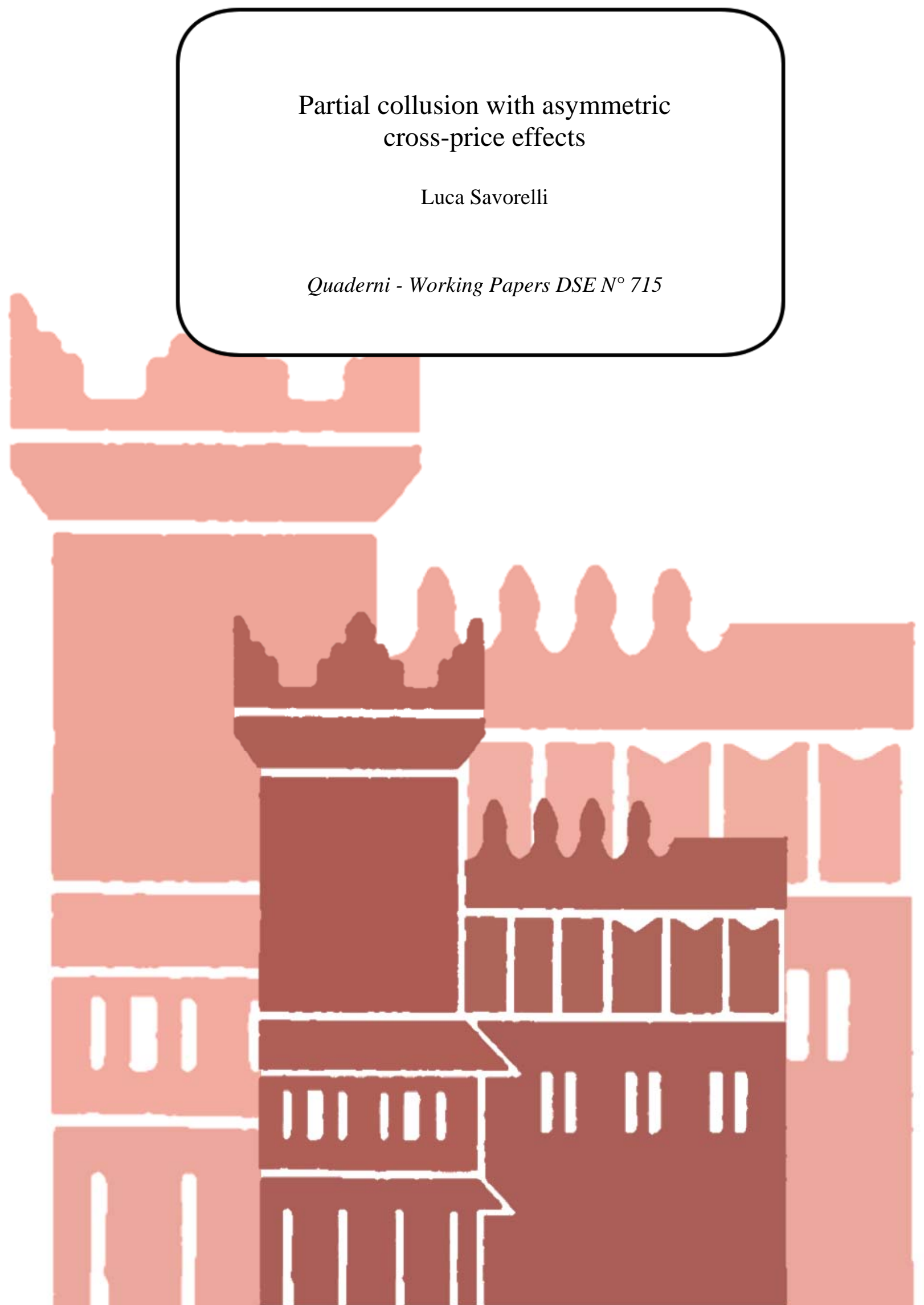




\title{
Partial collusion with asymmetric cross-price effects*
}

\author{
Luca Savorelli ${ }^{\dagger}$
}

October 8, 2010

\begin{abstract}
Asymmetries in cross-price elasticities have been demonstrated by several empirical studies. In this paper we study from a theoretical stance how introducing asymmetry in the substitution effects influences the sustainability of collusion. We characterize the equilibrium of a linear Cournot duopoly with substitute goods, and consider substitution effects which are asymmetric in magnitude. Within this framework, we study partial collusion using Friedman (1971) solution concept. Our main result shows that the interval of quantities supporting collusion in the asymmetric setting is always smaller than the interval in the symmetric benchmark. Thus, the asymmetry in the substitution effects makes collusion more difficult to sustain. This implies that previous Antitrust decisions could be reversed by considering the role of this kind of asymmetry.
\end{abstract}

Keywords: asymmetry, substitutes, Cournot duopoly, collusion, folk theorem.

JEL classification: C72, D43, L13.

\section{Introduction}

In this paper we study the sustainability of collusion in a Cournot duopoly where market demands are asymmetric in the magnitude of substitution effects. The evidence of asymmetric cross-price elasticities is shown in several empirical studies (e.g. Berry et al., 1995; Serthuraman et al., 1999; Kim and Cotterill, 2008; Rojas and Peterson, 2008). For example, in Berry et al. (1995) the cross-price semi-elasticity between Nissan Sentra and Ford

\footnotetext{
${ }^{*}$ The author is grateful to his $\mathrm{PhD}$ supervisor Vincenzo Denicolò, Fabio Pinna, Philipp Schimdt-Dengler, Pasquale Schiraldi, and the seminar's audience at the London School of Economics for their useful comments and suggestions. The author is also grateful to STICERD - London School of Economics and Political Science, where part of this work has been developed in his year as visiting research student.

${ }^{\dagger}$ Dipartimento di Scienze Economiche, Università di Bologna, piazza Scaravilli 1, 40126 Bologna, Italia; and Dipartimento di Economia Politica, piazza S. Francesco 7, 53100, Siena, Italia; e-mail: luca.savorelli@unibo.it.
} 
Escort is 1.375, while between Ford Escort and Nissan Sentra is 8.024; in the US market for processed cheese Kim and Cotterill (2008) find that the cross-price elasticity between Weight Watchers and Kraft is 0.25, while between Kraft and Weight Watchers is 0.04; the cross-price elasticity between Bud and Old Style is 0.003, while the cross-price elasticity between Old Style and Bud is 0.242 (Rojas and Peterson, 2008); finally, in a meta-analysis of 1060 cross-price effects, 19 grocery product categories and 280 brands, Serthuraman et al. (1999) provide an empirical generalization of this asymmetric price effect. The evidence supports the idea that in general cross-price elasticities are not symmetric, and this fact is also compatible with a theoretical perspective, since aggregate market demands need not satisfy any symmetry condition (see e.g. Diewert, 1980; Bonfrer et al. 2006), and also at the individual level the Slutsky matrix need not be symmetric (because the income effect need not be symmetric).

In this paper we consider a linear duopoly model with Cournot competition and substitute products. We extend Singh and Vives (1984) by allowing for asymmetry in the magnitude of the substitution effects, deriving the equilibrium quantities, prices and profits. We compare them to those in a symmetric equivalent duopoly setting, namely a duopoly in which the substitution parameters are symmetric and equal to the average of the parameters in the asymmetric case. Then, using the folk theorem solution concept and the penal code according to Friedman (1971), we analyze partial collusion. We derive the range of collusive quantities that makes the collusion sustainable for both firms, and compare it to the symmetric equivalent case.

This paper adds the two following main contributions to the existing literature. First, even though an extensive literature on collusion considers other kinds of asymmetries in market demands and in the characteristics of firms (for an overview see e.g. Feuerstein, 2005), to the best of our knowledge, the effect of asymmetric cross-price effects on the stability of collusion has not yet been studied from a theoretical stance. Our main result shows that, given the discount rate of each firms, the interval of quantities which supports the collusion is always smaller than in the symmetric equivalent case. Intuitively, if firms are asymmetric the intervals of the quantities making the collusion stable are no longer coincident, and the effect of asymmetry is to shift each firm's interval in opposite directions. This happens since a high collusion quantity raises the relative value of the deviation strategy for the weak firm, whose production decision is relatively more influenced by the other. Similarly, a low level of the collusion quantity makes the deviating behavior more convenient for the strong firm. Only the intermediate levels of collusive quantity are therefore supported by both firms. In this stylized setting, we thus conclude that the asymmetry in the substitution effect makes collusion more difficult to sustain. This 
implies that previous antitrust decisions could be reversed by considering the role of this kind of asymmetry.

The second result is related to the characterization of the equilibrium in the asymmetric Cournot duopoly. We find that the firm whose price is relatively more influenced by the other firm's production decisions sets a lower quantity, and sells at a lower price with respect to the rival, getting lower profits. An intuition for this result is that, since the two goods are strategic substitutes (but asymmetrically) the production decisions are driven by the firm which is relatively less influenced by the rival. The symmetric equivalent firm equilibrium prices, profits and quantities lie between those of the strong and the weak firm.

The paper is structured as follows. In the next section we characterize the equilibrium condition for the asymmetric Cournot duopoly and compare it to the symmetric equivalent case. In the third section we derive the solution of the collusion supergame, and we state the results on the implicit collusion stability. In the fourth section we draw the final remarks and directions for future theoretical and empirical research. The proofs are relegated to the appendix.

\section{A linear Cournot duopoly with asymmetric substitution effects}

In this section we consider a Cournot duopoly which extends Singh and Vives (1984) allowing for asymmetry in the magnitude of the substitution effects. This extension does not need any particular assumption, since market demands need not satisfy any symmetry conditions such as those required by the Slutsky equation (see e.g. Diewert, 1980; Bonfrer et al., 2006). Moreover, if the income effect in the Slutsky equation is not symmetric, the individual demands are not symmetric in the cross-price effect as well. Mastroleo and Savorelli (2010) show which class of individual utility functions underlies the asymmetry in cross substitution effects.

Let us consider a duopoly where firm $i$ and firm $j$ face the following inverse demand function

$$
p_{i}=a-q_{i}-b_{i} q_{j},\{i, j\}=\{1,2\}
$$

where $b_{i} \in[0,1], a>0$ and $q_{i, j} \geq 0$. We normalize the own-price effect to one, but this is not going to affect the results qualitatively. If $b_{i} \neq b_{j}$, we say that the demands are asymmetric, and if $b_{i}=b_{j}$ we say that they are symmetric. In a Cournot competition 
setting, each firm chooses the production quantity to maximize its profits as follows:

$$
\max _{q_{i}} \pi_{i}=\left(p_{i}-c\right) q_{i}
$$

where $c$ is a constant marginal cost. The solution of the model leads to the equilibrium quantities and profits

$$
\begin{aligned}
q_{i}^{C} & =\frac{\left(b_{i}-2\right)(a-c)}{b_{i} b j-4} \\
\pi_{i}^{C} & =\frac{\left(b_{i}-2\right)^{2}(a-c)^{2}}{\left(b_{i} b j-4\right)^{2}} \\
\Pi^{C} & =\pi_{i}+\pi_{j}=\frac{\left[\left(b_{i}-2\right)^{2}+\left(b_{j}-2\right)^{2}\right](a-c)^{2}}{\left(b_{i} b j-4\right)^{2}} .
\end{aligned}
$$

We assume henceforth that $a>c$ to guarantee that the Cournot equilibrium quantities are positive. To have an intuition about the strategic interaction driving the results, let us consider the following best response functions:

$$
\begin{aligned}
q_{i}^{C} & =\frac{\left(a-c-b_{i} q_{j}^{C}\right)}{2} \\
q_{j}^{C} & =\frac{\left(a-c-b_{j} q_{i}^{C}\right)}{2} .
\end{aligned}
$$

By simple inspection it is immediately apparent that the two goods are strategic substitutes. Then, taking the cross derivative of each best response function leads to the following Remark.

Remark 1 Consider $\frac{d q_{i}^{C}}{d q_{j}^{C}}=-\frac{b_{i}}{2}$ and $\frac{d q_{j}^{C}}{d q_{i}^{C}}=-\frac{b_{j}}{2}$, then:

1. if $b_{i}>b_{j}, \frac{d q_{i}^{C}}{d q_{j}^{C}}<\frac{d q_{j}^{C}}{d q_{i}^{C}}$

2. if $b_{i}<b_{j}, \frac{d q_{i}^{C}}{d q_{j}^{C}}>\frac{d q_{j}^{C}}{d q_{i}^{C}}$.

The above Remark states that, if e.g. $b_{i}>b_{j}$, an expansion in firm $j$ production has an impact on $i$ 's quantity choice greater than the impact that an equivalent expansion by $i$ has on the quantity choice of $j$. For this reason, we call $i$ the weak substitute and $j$ the strong substitute (and vice versa if $b_{i}<b_{j}$ ). Consistently, the strong substitute produces a higher quantity and gets higher profits with respect to the weak. Henceforth, the equilibrium results associated with the asymmetric demands will be denoted by the superscript $A S Y$. 
To allow for a comparison with the asymmetric setting, we exploit as benchmark a special case of the symmetric setting, such that $b_{i}=b_{j}=\frac{b_{i}+b_{j}}{2}$. The substitution parameters are chosen to be the mean of the parameters in the asymmetric case. We call the benchmark setting a symmetric equivalent, and we denote the associated equilibrium values by the superscript $S E$. In the symmetric equivalent case the solution of problem 2 is

$$
\begin{aligned}
q_{i}^{S E} & =q_{j}^{S E}=\frac{2(a-c)}{4+b_{i}+b j} \\
\pi_{i}^{S E} & =\frac{4(a-c)^{2}}{\left(4+b_{i}+b_{j}\right)^{2}} .
\end{aligned}
$$

To provide an intuitive comparison, in Figure 1 we represent the asymmetric Cournot equilibrium and its symmetric equivalent in the space of quantities when $b_{j}>b_{i}$.

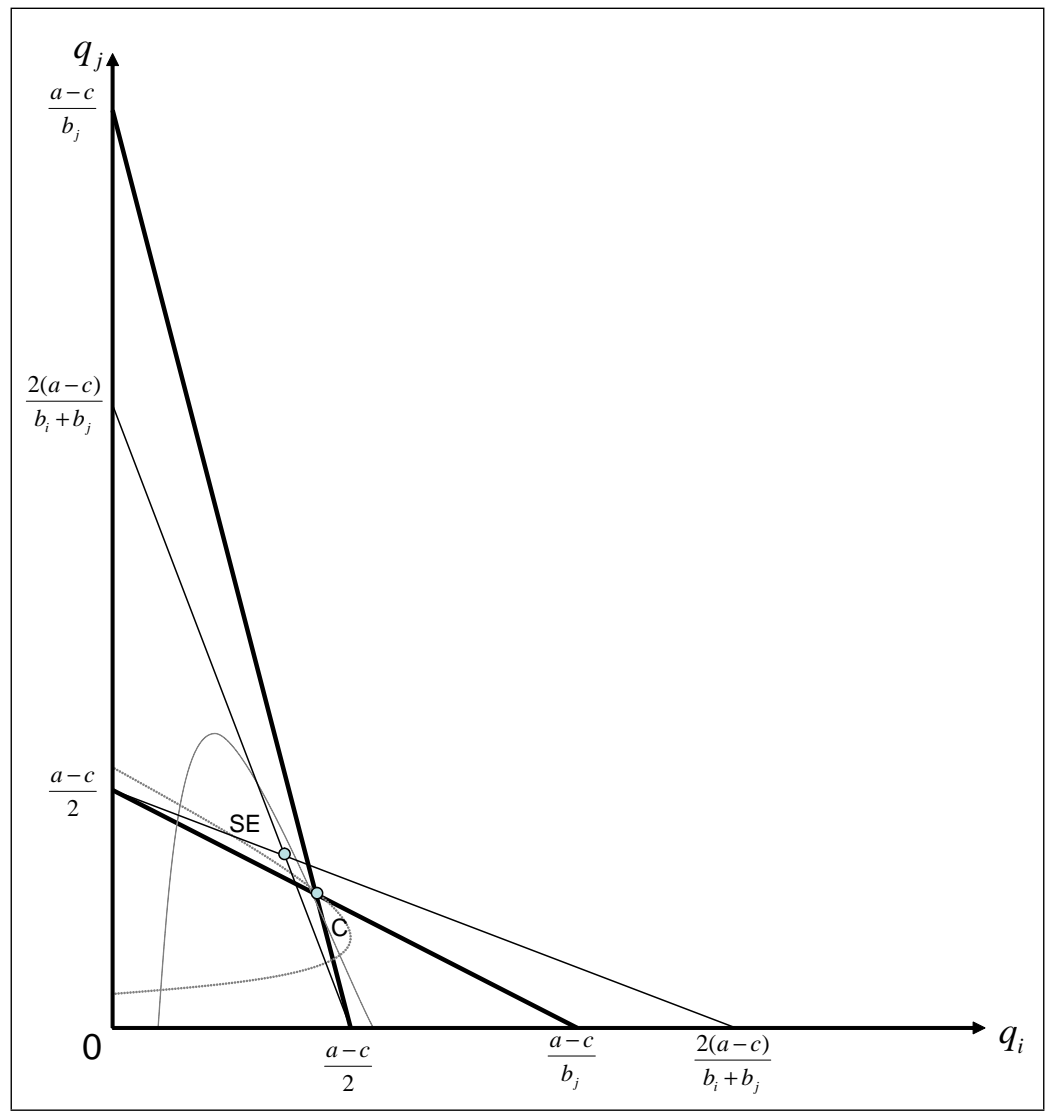

Figure 1

The best response functions are represented by the thick lines in the asymmetric case, and by the thin straight lines in the symmetric equivalent. The $S E$ equilibrium is North 
West of the asymmetric Cournot equilibrium, thus the weak substitute $j$ produces a lower quantity with respect to the $S E$ case, and the strong substitute $i$ a higher quantity. By considering the two parabolas, representing the contour lines of the profit functions at the Cournot equilibrium, it is straightforward to notice the asymmetry in the region of the possible equilibria which are Pareto superior to the Cournot-Nash.

The following Proposition characterizes the solution to problem 2 with respect to the equilibrium prices and the quantity levels, and compares them to the symmetric equivalent case.

Proposition 1 Let $q_{i}^{S E}=q_{j}^{S E}=q^{S E}$ and $p_{i}^{S E}=p_{j}^{S E}=p^{S E}$. Then the following holds:

$$
\begin{aligned}
& \text { 1. } b_{i}^{A S Y}>b_{j}^{A S Y} \Longleftrightarrow q_{i}^{A S Y}<q^{S E}<q_{j}^{A S Y} ; \\
& \text { 2. } b_{i}^{A S Y}>b_{j}^{A S Y} \Longleftrightarrow p_{i}^{A S Y}<p^{S E}<p_{j}^{A S Y} ; \\
& \text { 3. } b_{i}^{A S Y}>b_{j}^{A S Y} \Longleftrightarrow \pi_{i}^{A S Y}<\pi^{S E}<\pi_{j}^{A S Y} \text {. }
\end{aligned}
$$

The above Proposition states that in the symmetric equivalent case quantities, prices, and profits lie between the weak substitute's and the strong substitute's. The strategic weakness thus leads to lower quantities, prices and profits with respect to both the symmetric equivalent and the strong substitute.

\section{Partial collusion}

In this section we will investigate how asymmetry in substitutability affects the stability of collusion. As in the standard symmetric case, the implicit level of collusion should not necessarily be the monopoly quantity. The reason is that there are infinite quantities higher than the monopoly one that still provide profits greater than in the Cournot game. Moreover, as we will show in Remark 3, when the discount rate is sufficiently high the monopoly quantity does not make the collusion stable. Given a common discount factor

$\delta$, we study the minimum individual quantity produced by a firm that allows collusion to be stable, and to what extent asymmetry influences the stability of collusion.

We will proceed through the following steps. First, we will set up the Cournot supergame and study the collusion and deviation strategies, finding the interval of quantities for each firm that allows collusion to be sustainable. Second, we will state in Proposition 2 which is the interval of collusive quantities that makes collusion sustainable for both. Finally, in Proposition 3 we will state our main result, showing whether asymmetry in 
the substitution effects makes collusion easier with respect to the symmetric equivalent benchmark.

Over an infinite horizon, the two firms play grim trigger strategies in a Cournot supergame $\Gamma(\infty)$. We use the folk theorem solution concept according to Friedman (1971). In the first stage of the game, $t=0$, the firms follow a collusive strategy $\sigma^{*}$ and maximize the joint profits. In general, profits division is not equal. In the remaining time horizon, if both the firms played the collusive strategy in the previous period, the firms continue to play the collusive strategy $\sigma^{*}$. Otherwise, if at least one firm deviates from the collusive strategy playing $\sigma^{D}$, the firms play the Cournot-Nash strategy $\sigma^{C}$.

We first consider the collusive strategy $\sigma^{*}$. Since there are infinite potential collusive outcomes, we use joint-profit maximization as the selection criterion for the collusive focal point (as in e.g. d'Aspremont et al., 1983; and in asymmetric environments, e.g. Rothschild, 1999). Accordingly, we solve the following problem:

$$
\max _{q_{i}, q_{j}} \pi_{i}+\pi_{j}
$$

which leads to the equilibrium quantities and profits:

$$
\begin{aligned}
q_{i}^{*} & =q_{j}^{*}=\frac{a-c}{2+b_{i}+b j} \\
\pi_{i}^{*} & =\frac{\left(b_{j}+1\right)(a-c)^{2}}{\left(2+b_{i}+b_{j}\right)^{2}} .
\end{aligned}
$$

We can then state the following remark, which characterizes the joint-profit maximization equilibrium.

Remark 2 When firm $i$ and firm $j$ maximize the joint profit, they produce the same quantity, and the weak substitute obtains a level of profit lower than the strong.

The intuition for this Remark is that, since the two firms are technologically symmetric, when maximizing the joint profit they take into account the strategic externality deriving from the asymmetry in the strategic substitutability, and internalize it by playing like symmetric firms. Thus, it seems reasonable for this equilibrium to be a focal point also for lower levels of joint profits.

When the firms play the $\sigma^{*}$ strategy, each firm gets the profits $\pi_{i}^{*}=q_{i}^{*}\left(a-c-q_{i}^{*}-b_{i} q_{j}^{*}\right)$. Taking into account the characterization in Remark 2 , the firms set $q_{i}^{*}=q_{j}^{*}$. We can therefore rewrite the above expression as 


$$
\pi_{i}^{*}=q_{j}^{*}\left(a-c-\left(1+b_{i}\right) q_{j}^{*}\right) .
$$

Notice again that with asymmetric demands the collusive quantities are still symmetric, but the profits are not.

We now derive the equilibrium values for firm $i$ playing $\sigma^{D}$, denoting the solutions with the superscript $D$. In the period of deviation, firm $i$ solves the following maximization problem:

$$
\max _{\left\{q_{i}^{D} \mid q_{j}=q_{j}^{*}\right\}} \pi_{i}=\left(p_{i}-c\right) q_{i}=q_{i}^{D}\left(a-c-q_{i}^{D}-b_{i} q_{j}^{*}\right)
$$

and obtains the following per-period quantities and profits:

$$
\begin{aligned}
q_{i}^{D} & =\frac{\left(a-c-b_{i} q_{j}^{*}\right)}{2} \\
\pi_{i}^{D} & =\frac{\left(a-c-b_{i} q_{j}^{*}\right)^{2}}{4} .
\end{aligned}
$$

Thus, the flow of profits for the deviating firm $i$ is

$$
\Phi_{i}^{D}=\pi_{i}^{D}\left(q_{j}^{*}\right)+\frac{\delta}{1-\delta} \pi_{i}^{A S Y}
$$

where $\delta \in[0,1]$ is the discount rate common to $i$ and $j$.

In what follows we will study the problem of collusion stability. We will first derive the interval of quantities of the rival firm that makes the collusion sustainable, and then the interval of quantities on which both the firms agree to collude.

For firm $i$, the collusive strategy is sustainable only if $\Phi_{i}^{D} \leq \pi_{i}^{*}\left(q_{j}^{*}\right) /(1-\delta)$. Then, the following lemma states for which values $q_{i, j}^{*}$ the collusion is stable for each single firms.

Lemma 1 The collusion is stable for firm $i$ if and only if $\underline{q}_{j}^{\text {Col }}<q_{j}^{*}<\bar{q}_{j}^{C o l}$, and for firm $j$ if and only if $\underline{q}_{i}^{C o l}<q_{i}^{*}<\bar{q}_{i}^{C o l}$, where:

$$
\begin{aligned}
\bar{q}_{i}^{C o l} & =(a-c) \frac{\left(b_{i} b_{j}-4\right)\left[b_{i}(\delta-1)-2\right]+A_{i}}{\left(b_{i} b_{j}-4\right)\left[\delta b_{i}^{2}-\left(2+b_{i}\right)^{2}\right]} \\
\underline{q}_{i}^{C o l} & =(a-c) \frac{\left(b_{i} b_{j}-4\right)\left[b_{i}(\delta-1)-2\right]-A_{i}}{\left(b_{i} b_{j}-4\right)\left[\delta b_{i}^{2}-\left(2+b_{i}\right)^{2}\right]} \\
\bar{q}_{j}^{C o l} & =(a-c) \frac{\left(b_{i} b_{j}-4\right)\left[b_{j}(\delta-1)-2\right]+A_{j}}{\left(b_{i} b_{j}-4\right)\left[\delta b_{j}^{2}-\left(2+b_{j}\right)^{2}\right]} \\
\underline{q}_{j}^{C o l} & =(a-c) \frac{\left(b_{i} b_{j}-4\right)\left[b_{j}(\delta-1)-2\right]-A_{j}}{\left(b_{i} b_{j}-4\right)\left[\delta b_{j}^{2}-\left(2+b_{j}\right)^{2}\right]}
\end{aligned}
$$


and $A_{i}=\left[\delta b_{i}\left(\delta b_{i}\left(b_{i}-2\right)^{2}+b_{i}\left(b_{i}^{2}-b_{j}^{2}\right)+8\left(b_{i}-b_{j}\right)\right)\right]^{\frac{1}{2}}, A_{j}=\left[\delta b_{j}\left(\delta b_{j}\left(b_{j}-2\right)^{2}+b_{j}\left(b_{j}^{2}-\right.\right.\right.$ $\left.\left.\left.b_{i}^{2}\right)+8\left(b_{j}-b_{i}\right)\right)\right]^{\frac{1}{2}}$.

Henceforth we will consider only real-valued boundaries quantities ${ }^{1}$ We call $Q_{i}=$ $\left\{\underline{q}_{i}^{C o l}<q_{i}^{*}<\bar{q}_{i}^{C o l}\right\}$ and $Q_{j}=\left\{\underline{q}_{j}^{C o l}<q_{j}^{*}<\bar{q}_{j}^{C o l}\right\}$ the sets of sustainable collusion quantities for firm $i$ and $j$. As it is evident by inspection, the two interval do not coincide as long $b_{i} \neq b_{j}$. Some collusive quantities are thus sustainable for firm $i$, but not for firm $j$. Since it could also be the case that the two intervals do not overlap, in the following Lemma we state when there is room for collusion among firms if the two firms are asymmetric.

Lemma 2 If $b_{i} \neq b_{j}$, then $Q_{i} \neq Q_{j}$ and $Q_{i} \cap Q_{j}$ always exists.

The above Lemma means that there is always an interval of quantities on which the two firms can agree to collude, and which is sustainable for both. Let us call $Q^{A S Y}=Q_{i} \cap Q_{j}$ the set of collusive quantities which are sustainable for both firm $i$ and firm $j$. By contrast, when $b_{i}=b_{j}, \underline{q}_{i}^{C o l}=\underline{q}_{j}^{C o l}=\underline{q}^{C o l}$ and $\bar{q}_{i}^{C o l}=\bar{q}_{j}^{C o l}=\bar{q}^{C o l}$, and the two sets coincide. In this case, we call the set of collusive quantities $Q^{S Y M}$. The following proposition characterizes the set of collusive quantities that makes the collusion stable for each case.

\section{Proposition 2 When:}

$$
\begin{aligned}
& \text { 1. } b_{i}<b_{j} \Leftrightarrow Q^{A S Y}=\left\{\underline{q}_{j}^{C o l}<q_{i, j}^{*}<\bar{q}_{i}^{C o l}\right\} ; \\
& \text { 2. } b_{i}=b_{j} \Leftrightarrow Q^{S Y M}=\left\{\underline{q}^{C o l}<q^{*}<\bar{q}^{C o l}\right\} ; \\
& \text { 3. } b_{i}>b_{j} \Leftrightarrow Q^{A S Y}=\left\{\underline{q}_{i}^{C o l}<q_{i, j}^{*}<\bar{q}_{j}^{C o l}\right\} .
\end{aligned}
$$

First, notice that setting $b_{1}=b_{2}=1$, the boundary values in the above Lemma reduce to the well known symmetric case

$$
\frac{(9-5 \delta)(a-c)}{3(9-\delta)}<q_{i, j}^{*}<\frac{a-c}{3} .
$$

Second, setting $\delta=0$, the boundaries of the stable collusion quantities are $\left[\frac{a-c}{2+b_{j}}, \frac{a-c}{2+b_{i}}\right]$ in case 1 , and the reverse in case 2 . The lower boundary is thus greater than the collusive monopoly quantity, $\frac{a-c}{2+b_{j}+b_{i}}$. This leads to the following remark.

\footnotetext{
${ }^{1}$ When $b_{i}>b_{j}, A_{i}$ is always positive and $A_{j}$ is positive when $\frac{\left(8 b_{i}-\left(8+b_{i}^{2}\right) b_{j}+b_{j}^{3}\right)}{\left(\left(b_{j}-2\right)^{2} b_{j}\right)}<d$ and, in addition, to be real valued, if $b_{j}<\frac{13-\sqrt{41}}{8} \simeq 0,825$, it must be $b_{i}<\frac{2\left(2-\left[\left(b_{j}-2\right)^{2}\left(b_{j}+1\right)\right]^{\frac{1}{2}}\right)}{b_{j}}$. When $b_{i}<b_{j}, A_{j}$ is always positive and analogous conditions can be obtained by inverting indexes.
} 
Remark 3 There always exist a value $0 \leq \delta \leq 1$ such that the collusive monopoly equilibrium is not stable.

The above remark points out that, as in the symmetric case, the monopoly quantity is not always a feasible quantity, and when this is the case the analysis of partial collusion thus becomes more relevant.

We proceed to answer the key research question of this paper, i.e. whether asymmetry in the substitution effects makes collusion more difficult to sustain. Without qualitatively affecting our results, we normalize the parameter space by setting $b_{j}=1-b_{i}$. We define the index of asymmetry as $\gamma=\left|b_{j}-b_{i}\right|=\left|1-2 b_{i}\right|$. When the two values are symmetric, $b_{i}=b_{j}=\frac{1}{2}$ and $\gamma=0$, while $\gamma=1$ when one parameter is 0 and the other 1 , the maximum level of asymmetry. We then use the average value of the parameter as benchmark, and we state the main result of this paper in the following proposition.

Proposition 3 The interval of collusive quantities in the symmetric equivalent case is always larger than the one in the asymmetric case.

The intuition behind this proposition can be understood by highlighting the strategic interaction between the two firms. Each firm determines what is the level of production of the other firm that makes the collusion stable for itself. When firms collude, it is optimal for both to produce the same quantity. Then, it can immediately be seen from (13) that, if the weak substitute wants to deviate from the agreement, he is going to produce a lower deviation quantity with respect to the deviation quantity of the strong substitute. The Cournot quantity in the following period is lower as well. The reason is that a high collusion quantity raises the relative value of the deviation strategy for the weak substitute. Analogously, a low level of the collusion quantity makes the deviating behavior more convenient for the strong substitute. The asymmetry in the strategic substitution effect translates into asymmetric partial collusion strategies, which still overlap, but are no longer coincident. Each firm's collusive interval is shifted in opposite directions, and only the intermediate levels of collusive quantity are supported by both firms. We can therefore conclude that the asymmetry in the substitution effect makes collusion more difficult to sustain with respect to the symmetric benchmark case.

\section{Conclusions}

In this paper we generalized Singh and Vives (1984) to account for asymmetry in the substitution effects, and to study its implications for implicit collusion. The first result 
we found characterizes Cournot equilibrium: The symmetric benchmark firm equilibrium prices, profits and quantities are lower than those of the strong, and higher than those of the weak. The second result states that the asymmetry in the substitution effects makes partial collusion more difficult to support with respect to its symmetric benchmark.

Future extensions of this model could explore the robustness of the results under different settings and kinds of competition (e.g. Bertrand competition, semi-collusion, R\&D). Our feeling is that, in frameworks other than collusion, introducing asymmetries in the cross-price effect could lead to new theoretical insights particularly useful to empirical analysis.

Finally, this paper suggests that the asymmetry in the substitution effects is a relevant issue when evaluating the possibility of implicit collusion among firms. If the empirical estimations of market demands do not take into account this kind of asymmetry, it is likely that the extent to which the firms can collude is overestimated. We thus think that there is room for an empirical re-assessment of previous estimations, and that in this light perhaps some anti-trust decisions could be reversed. Moreover, the theory proposed in this paper could be usefully tested by using experimental economics methodology.

\section{Appendix}

\subsection{Proof of Proposition 1}

Part 1. By simple inspection, as long as $b_{i}^{A S Y}>b_{j}^{A S Y}$ we know that $q_{i}^{A S Y}<q_{j}^{A S Y}$. Then it is sufficient to solve the inequalities $q_{i}^{A S Y}<q^{S E}$ and $q^{S E}<q_{j}^{A S Y}$ for value of the parameters of the problem, that is $b_{i, j} \in[0,1]$ and $a>c>0$. Part 2. From the solutions of the symmetric and asymmetric problem it is simple to get $p_{i}^{A S Y}=\frac{a\left(b_{i}-2\right)+\left[b_{i}\left(b_{j}-1\right)\right] c-2}{\left(b_{i} b_{j}-4\right)}, p^{S E}=$ $\frac{2 a+\left(2+b_{i}+b_{j}\right) c}{4+b_{i}+b_{j}}, p_{j}^{A S Y}=\frac{a\left(b_{j}-2\right)+\left[b_{j}\left(b_{i}-1\right)\right] c-2}{\left(b_{i} b_{j}-4\right)}$ and analogously to Part 1 solving the corresponding inequalities. Part 3. The solution to the inequality is analogous and straightforward by considering 8 and 4 .

\subsection{Proof of Lemma 1}

Let us consider the inequalities $\Phi_{i}^{D} \leq \pi_{i}^{*}\left(q_{j}^{*}\right) /(1-\delta)$, that is:

$$
\begin{gathered}
\pi_{i}^{D}\left(q_{j}^{*}\right)+\frac{\delta}{1-\delta} \pi_{i}^{A S Y} \leq \frac{\pi_{i}^{*}\left(q_{j}^{*}\right)}{1-\delta} \\
\frac{\left(a-c-b_{i} q_{j}^{*}\right)^{2}}{4}+\frac{\delta}{1-\delta} \frac{\left(b_{i}-2\right)^{2}(a-c)^{2}}{\left(b_{i} b j-4\right)^{2}} \leq \frac{q_{j}^{*}\left(a-2 q_{j}^{*}-c\right)}{1-\delta}
\end{gathered}
$$


The solution of the associated equation gives the two values

$$
\begin{aligned}
\bar{q}_{i}^{C o l} & =(a-c) \frac{\left(b_{i} b_{j}-4\right)\left[b_{i}(\delta-1)-2\right]+A_{i}}{\left(b_{i} b_{j}-4\right)\left[\delta b_{i}^{2}-\left(2+b_{i}\right)^{2}\right]} \\
\underline{q}_{i}^{C o l} & =(a-c) \frac{\left(b_{i} b_{j}-4\right)\left[b_{i}(\delta-1)-2\right]-A_{i}}{\left(b_{i} b_{j}-4\right)\left[\delta b_{i}^{2}-\left(2+b_{i}\right)^{2}\right]}
\end{aligned}
$$

where

$A_{i}=\left[\delta b_{i}\left(\delta b_{i}\left(b_{i}-2\right)^{2}+b_{i}\left(b_{i}^{2}-b_{j}^{2}\right)+8\left(b_{i}-b_{j}\right)\right)\right]^{\frac{1}{2}}$.

Inverting the indexes we obtain analogous values for firm $j$, that is

$$
\begin{aligned}
\bar{q}_{j}^{C o l} & =(a-c) \frac{\left(b_{i} b_{j}-4\right)\left[b_{j}(\delta-1)-2\right]+A_{j}}{\left(b_{i} b_{j}-4\right)\left[\delta b_{j}^{2}-\left(2+b_{j}\right)^{2}\right]} \\
\underline{q}_{j}^{C o l} & =(a-c) \frac{\left(b_{i} b_{j}-4\right)\left[b_{j}(\delta-1)-2\right]-A_{j}}{\left(b_{i} b_{j}-4\right)\left[\delta b_{j}^{2}-\left(2+b_{j}\right)^{2}\right]}
\end{aligned}
$$

where

$$
A_{j}=\left[\delta b_{j}\left(\delta b_{j}\left(b_{j}-2\right)^{2}+b_{j}\left(b_{j}^{2}-b_{i}^{2}\right)+8\left(b_{j}-b_{i}\right)\right)\right]^{\frac{1}{2}} .
$$

We want the interval of collusive quantities for both firm $i$ and $j$ to be real valued. When $b_{i}>b_{j}, A_{i}$ 's radicand is always positive and $A_{j}$ 's is positive when $\frac{\left(8 b_{i}-\left(8+b_{i}^{2}\right) b_{j}+b_{j}^{3}\right)}{\left(\left(b_{j}-2\right)^{2} b_{j}\right)}<$ $d$ and, in addition, if $b_{j}<\frac{13-\sqrt{41}}{8} \simeq 0,825$, it must be $b_{i}<\frac{2\left(2-\left[\left(b_{j}-2\right)^{2}\left(b_{j}+1\right)\right]^{\frac{1}{2}}\right)}{b_{j}}$. When $b_{i}<b_{j}, A_{j}$ is always positive and analogous conditions can be obtained by inverting indexes.

\subsection{Proof of Lemma 2}

Lemma 3 If $b_{i} \neq b_{j}$, then $Q_{i} \neq Q_{j}$ and $Q_{i} \cap Q_{j}$ always exists.

We will first show that, when $b_{i}>b_{j} \Longleftrightarrow \underline{q}_{j}^{C o l}<\underline{q}_{i}^{C o l}<\bar{q}_{j}^{C o l}<\bar{q}_{i}^{C o l}$; the second part of the proof showing $b_{i}<b_{j} \Longleftrightarrow \underline{q}_{i}^{C o l}<\underline{q}_{j}^{C o l}<\bar{q}_{i}^{C o l}<\bar{q}_{j}^{C o l}$ is analogous, and can be obtained by inverting the indexes.

Let us consider $b_{i}>b_{j}$. We first prove that $\underline{q}_{j}^{C o l}<\bar{q}_{j}^{C o l}$. This can be done solving the inequality

$$
(a-c) \frac{\left(b_{i} b_{j}-4\right)\left[b_{j}(\delta-1)-2\right]-A_{j}}{\left(b_{i} b_{j}-4\right)\left[\delta b_{j}^{2}-\left(2+b_{j}\right)^{2}\right]}<(a-c) \frac{\left(b_{i} b_{j}-4\right)\left[b_{j}(\delta-1)-2\right]+A_{j}}{\left(b_{i} b_{j}-4\right)\left[\delta b_{j}^{2}-\left(2+b_{j}\right)^{2}\right]}
$$


considering the relevant range of the parameters $a, b_{i}, b_{j}, c, \delta$, and the conditions stated in Lemma 1 for the quantities to be real-valued, this inequality always holds. Then, we prove that $\underline{q}_{i}^{C o l}<\bar{q}_{i}^{C o l}$. The following inequality

$$
(a-c) \frac{\left(b_{i} b_{j}-4\right)\left[b_{i}(\delta-1)-2\right]-A_{i}}{\left(b_{i} b_{j}-4\right)\left[\delta b_{i}^{2}-\left(2+b_{i}\right)^{2}\right]}<(a-c) \frac{\left(b_{i} b_{j}-4\right)\left[b_{i}(\delta-1)-2\right]+A_{i}}{\left(b_{i} b_{j}-4\right)\left[\delta b_{i}^{2}-\left(2+b_{i}\right)^{2}\right]}
$$

always holds, considering as above the relevant range of the parameters and the condition for real-values quantities. Finally, to ensure that the two intervals always overlap, we prove that $\underline{q}_{j}^{C o l}<\bar{q}_{i}^{C o l}$, that is

$$
(a-c) \frac{\left(b_{i} b_{j}-4\right)\left[b_{j}(\delta-1)-2\right]+A_{j}}{\left(b_{i} b_{j}-4\right)\left[\delta b_{j}^{2}-\left(2+b_{j}\right)^{2}\right]}<(a-c) \frac{\left(b_{i} b_{j}-4\right)\left[b_{i}(\delta-1)-2\right]-A_{i}}{\left(b_{i} b_{j}-4\right)\left[\delta b_{i}^{2}-\left(2+b_{i}\right)^{2}\right]}
$$

always holds. The remaining inequalities can be derived by transitivity.

\subsection{Proof of Proposition 2}

Proposition 2 follows directly by Lemma 1 and 2 . The two asymmetric firms can agree on the collusive quantity belonging to the interval where the two individual partial collusion strategies overlap.

\subsection{Proof of Proposition 3}

Let us normalize the parameter space by setting $b_{j}=1-b_{i}$. Then, consider $b_{i}<b_{j}$. By Proposition 2, $\underline{q}_{i}^{C o l}<q_{i, j}^{*}<\bar{q}_{j}^{C o l}$ and, since assuming positive real-valued outputs, we call $l^{A S Y}=\bar{q}_{j}^{C o l}-\underline{q}_{i}^{C o l}$ the length of the set of collusive quantities in the asymmetric case, i.e.

$l^{A S Y}=(a-c) \frac{-\left(4+b_{i}\left(b_{i}-1\right)\right)^{2}\left[2(\delta-15)+b_{i}\left(-5+2 \delta b_{i}^{2}(\delta-1)+\delta(12+\delta)+b_{i}(5-3 \delta(2+\delta))\right]+2\left(4-b_{i}+b_{i}\right)\left[B_{1}+B_{2}\right]\right.}{\left[4+b_{i}\left(b_{i}-1\right)\right]^{2}\left[\left(b_{i}-3\right)^{2}+\delta\left(b_{i}-1\right)^{2}\right]\left[\delta b_{i}^{2}-\left(2+b_{i}\right)^{2}+\delta b_{i}^{2}\right]}$ where:

$B_{1}=\left[\delta b_{i}\left(b_{i}\left(17-2 b_{i}+\delta\left(b_{i}-2\right)^{2}-8\right)\right)\right]^{\frac{1}{2}}$,

and

$B_{2}=\left[\delta\left(7+\delta+b_{i}\left(b_{i}\left(11+2 b_{i}+\left(b_{i}-2\right) \delta\right)-20\right)\right]^{\frac{1}{2}}\right.$.

In the standard equivalent case, when $b_{i}=b_{j}=\frac{1}{2}$, the interval $l^{S E}=\bar{q}-\underline{q}$ is given by

$$
l^{S E}=(a-c) \frac{\delta 16}{5(\delta-25)} .
$$

Then, considering the inequality $l^{A S Y}>l^{S E},(a-c)$ cancel out and the resulting inequality is verified for all the values of the parameters of the problem $\left(b_{i}, b_{j}, \delta\right)$ and it is independent also from the calibration of $a$ and $c$. 
The case in which $b_{i}<b_{j}$ can be proved analogously by considering the length $l^{A S Y}=$

$\bar{q}_{i}^{C o l}-\underline{q}_{j}^{C o l}$ and comparing it to $l^{S E}$. The results and the proof are analogous, and can be obtained simply by inverting indexes.

\section{References}

Berry, S., J. Levinsohn, and A. Pakes (1995). Automobile prices in market equilibrium. Econometrica: Journal of the Econometric Society 63(4), 841-890.

Bonfrer, A., E. Berndt, and A. Silk (2006). Anomalies in Estimates of Cross-Price Elasticities for Marketing Mix Models: Theory and Empirical Test. NBER Working Paper.

D'Aspremont, C., A. Jacquemin, J. Gabszewicz, and J. Weymark (1983). On the stability of collusive price leadership. The Canadian Journal of Economics / Revue canadienne d'Economique 16(1), 17-25.

Diewert, W. (1980). Symmetry conditions for market demand functions. The Review of Economic Studies 47(3), 595-601.

Feuerstein, S. (2005). Collusion in industrial economics - A survey. Journal of Industry, Competition and Trade 5(3), 163-198.

Friedman, J. (1971). A non-cooperative equilibrium for supergames. The Review of Economic Studies 38(1), 1-12.

Kim, D. and R. Cotterill (2008). Cost pass-through in differentiated product markets: the case of us processed cheese. Journal of Industrial Economics 56(1), 32-48.

Mastroleo, M. and L. Savorelli (2009). Asymmetric substitutability and complementarity in individual demand. MIMEO.

Rojas, C. and E. Peterson (2008). Demand for differentiated products: Price and advertising evidence from the US beer market. International Journal of Industrial Organization 26(1), 288-307.

Rothschild, R. (1999). Cartel stability when costs are heterogeneous. International Journal of Industrial Organization 17(5), 717-734.

Sethuraman, R., V. Srinivasan, and D. Kim (1999). Asymmetric and neighborhood crossprice effects: some empirical generalizations. Marketing Science 18(1), 23-41. 
Singh, N. and X. Vives (1984). Price and quantity competition in a differentiated duopoly. The RAND Journal of Economics 15(4), 546-554. 


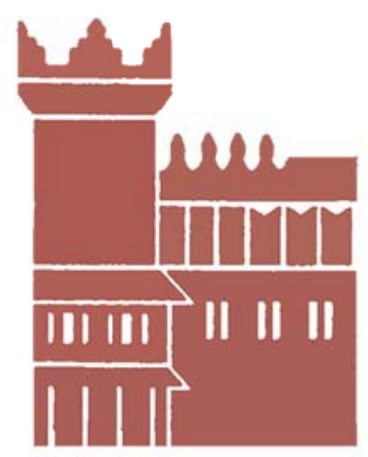

Alma Mater Studiorum - Università di Bologna DEPARTMENT OF ECONOMICS

Strada Maggiore 45

40125 Bologna - Italy

Tel. +39051 2092604

Fax +390512092664

http://www.dse.unibo.it 